\title{
Hemizona Assay and Sperm Penetration Assay in the Prediction of IVF Outcome: A Systematic Review
}

\author{
Paraskevi Vogiatzi, ${ }^{1}$ Charalampos Chrelias, ${ }^{2}$ David J. Cahill, ${ }^{3}$ Maria Creatsa, ${ }^{1}$ \\ Nikos Vrachnis, ${ }^{4}$ Zoe Iliodromiti, ${ }^{4}$ Demetrios Kassanos, ${ }^{2}$ and Charalampos Siristatidis ${ }^{1}$ \\ ${ }^{1}$ Assisted Reproduction Unit, 3rd Department of Obstetrics and Gynecology, University of Athens, Attikon Hospital, Rimini 1, \\ Chaidari, 12642 Athens, Greece \\ ${ }^{2} 3 r d$ Department of Obstetrics and Gynecology, University of Athens, Attikon Hospital, Rimini 1, Chaidari, 12642 Athens, Greece \\ ${ }^{3}$ School of Clinical Sciences, University of Bristol, Level D, St Michael's Hospital, Bristol BS2 8EG, UK \\ ${ }^{4}$ 2nd Department of Obstetrics and Gynecology, University of Athens, Aretaieion Hospital, Vasilissis Sofias 76, 11528 Athens, Greece
}

Correspondence should be addressed to Charalampos Siristatidis; harrysiri@yahoo.gr

Received 9 July 2013; Accepted 1 September 2013

Academic Editor: Benjamin N. Breyer

Copyright (C) 2013 Paraskevi Vogiatzi et al. This is an open access article distributed under the Creative Commons Attribution License, which permits unrestricted use, distribution, and reproduction in any medium, provided the original work is properly cited.

\begin{abstract}
The limited predictive value of semen analysis in achieving natural conception or in IVF outcome confirms the need for sperm function tests to determine optimal management. We reviewed HZA and SPA predictive power in IVF outcome, with statistical significance of diagnostic power of the assays. HZA was readily efficient in predicting IVF outcome, while evident inconsistency among the studies analysed framed the SPA's role in male fertility evaluation. Considerable variation was noted in the diagnostic accuracy values of SPA with wide sensitivity (52-100\%), specificity (0-100\%), and PPV (18-100\%) and NPV (0-100\%) together with fluctuation and notable differentiation in methodology and cutoff values employed by each group. HZA methodology was overall consistent with minor variation in cutoff values and oocyte source, while data analysis reported strong correlation between HZA results with IVF outcome, high sensitivity (75-100\%), good specificity (57-100\%), and high PPV (79-100\%) and NPV (68-100\%). HZA correlated well with IVF outcome and demonstrated better sensitivity/specificity and positive/negative predictive power. Males with normal or slightly abnormal semen profiles could benefit by this intervention and could be evaluated prior to referral to assisted reproduction. HZA should be used in a sequential fashion with semen analysis and potentially other bioassays in an IVF setting.
\end{abstract}

\section{Introduction}

Male fertility is considered to be affected by genetic disorders, congenital or acquired urogenital abnormalities, varicocele, infection, endocrine disturbances, immunological and/or lifestyle factors, environmental assaults, and idiopathic factors with no demonstrable aetiology [1]. Fifteen percent of all couples of reproductive age are affected by infertility [2], with male factor being solely responsible in about $20 \%$ of these and a contributory factor in another $30 \%-40 \%$ [3]. The latter together with the lasting debate over sperm quality and density decline [4-7] intensifies the need for accurate and rapid evaluation of sperm function in assisting clinical decisions, that is, whether natural conception is feasible or medical support should be sought for reproduction, taking the age of the woman and the duration of infertility into account.

Semen analysis is the basis of primary male investigation, and in certain cases, such as in severe oligozoospermia, semen parameters are of absolute importance and should be strongly considered in clinical management by implementing the World Health Organization (WHO) and the European Society of Human Reproduction and Embryology (ESHRE) guidelines and recommendations [8]. However, the limited predictive value of semen analysis in achieving natural conception or in assisted reproductive technology (ART) outcome such as in in vitro fertilization (IVF) [9-12] confirms the need for sperm function tests, as well as in cases of oligoasthenoteratozoospermia or idiopathic infertility when 
knowledge of sperm functional capacity is a requisite for determining optimal management.

The sperm penetration assay (SPA) was introduced by Yanagimachi et al. [13] and involved the use of surrogate ova from golden hamsters, stripped of the zona pellucida to allow interspecies interaction. SPA evaluates sperm function capacity by examining sperm competence in distinct biological processes required for fertilization; capacitation, acrosome reaction, spontaneous recognition of and fusion with vitelline membrane to the end-point of chromatin decondensation [13]. The outcome of this assay is hamster oocyte penetration rate expressed as the percentage of ova with positive penetration divided by the total number of ova, in control and test samples (supplemental Figure 1 (see supplemental Figure 1 in Supplemntary Material available online at http://dx.doi.org/10.1155/2013/945825)). Positive penetration is confirmed through the presence of swollen sperm head with a visible tail or male pronucleus, through microscopical examination of the ooplasm. Correspondingly, the concept of sperm-zona pellucida binding assay was first introduced by Liu et al. [14], who pioneered the competitive zona binding (CBZ) test, and Burkman et al. [15] who established the hemizona assay (HZA) the same year. Franken et al. [16] described salt storaging of human oocytes, while Morroll et al. [17] initiated the utilization of cryostored oocytes for this assay. HZA is a functional homologous model of gamete interaction that uses nonviable, bisected human oocytes to examine spermatozoa capacity to bind to glycoprotein receptors $\mathrm{ZP} 3 / \mathrm{ZP} 4$ on the zona surface and undergo activation and acrosome reaction in an appropriate timed manner. The main principle of this assay is the assessment of spermzona binding in vitro and its numerical interpretation by the calculation of the hemizona assay index (HZI) value (supplemental Figure 2). It has been estimated that defective spermzona binding and zona penetration are among the most common causes of fertilization failure and that within the oligozoospermic population around $80 \%$ of males produce spermatozoa that are unable to interact normally with zona pellucida [18-21]. Both SPA and HZA have the potential to represent male fertility indicators, as both techniques reflect certain biological requisites for reproduction; a sequence of functional conditions is required for the sperm to interact and fuse with the ovum.

The validity of any diagnostic test is based on a set of criteria according to ESHRE: sensitivity (to produce few falsenegatives), specificity (to produce few false-positives), complexity, time, and cost effectiveness, and positive (PPV) and negative (NPV) predictive values (in terms of fertility and fertilization rates) [9]. The aim of the present study was to review and appraise the published evidence to date on two major techniques: SPA and HZA. We reviewed their predictive power in IVF outcome and performed an assay overview in terms of protocol standardization, to determine whether these tests could be incorporated in routine male fertility assessment and whether they could be used as a tool for predicting fertilization potential.

\section{Materials and Methods}

2.1. Search Strategy. This systematic review was conducted according to the PRISMA guidelines [22] and the initial protocol agreed by all authors. Studies in English language from 1976 to August 2012 were compiled with no study design restrictions, using the following search algorithms in two major scientific/medical databases (Medline/PubMed, ScienceDirect):

(i) "sperm penetration assay" OR "SPA" OR "HEPT" OR “ZHEPT” OR “HEPA" OR "HOPT” OR "hamster test" AND "IVF" OR "in vitro fertilization" OR “in vitro fertilisation" OR “assisted reproduction" OR "Reproductive Techniques, Assisted” OR "ART”

(ii) "hemizona assay" OR "HZA" OR "sperm zona test" AND "IVF" OR "in vitro fertilization" OR "in vitro fertilisation" OR "assisted reproduction" OR "Reproductive Techniques, Assisted" OR "ART".

Search results were confirmed through the SCIRUS database. There were no MeSH terms assigned to the specific assays. Reference lists of relevant articles were hand-searched for potentially eligible studies.

2.2. Screening. To minimize bias (extraction, recording, conformity, and retrieval), three authors (P. Vogiatzi, C. Siristatidis, and M. Creatsa) performed the primary evaluation of titles and abstracts identified through the publication identification process, and each author provided a list of potentially eligible studies. Those three authors extracted the data independently, using a preagreed data extraction form. Collected data included general information (title, author, year, journal, and clinical setting), study characteristics (design, inclusions/exclusions), participants' characteristics (cause and duration of preexisting infertility, semen parameters, and protocols for ovarian stimulation), assay parameters (SPA/HZA and fertilization rate cutoff values, different culture media and sperm enhancers used, incubation lengths, hemizona sources, and preservation methods), and results (number of participants, reference population, specificity and sensitivity of the assay, and PPV and NPV as reported or calculated by the data sets provided by the study group). If multiple publications using the same cohort were identified, the most recent or more complete publication was used for data extraction. Two authors (P. Vogiatzi and C. Siristatidis) performed the final selection of the potential eligible studies; conflicts were resolved by team consensus.

2.3. Eligibility. Studies comparing HZA and SPA results with outcome parameters in an IVF setting were considered to examine the validity of these tests. Studies had to be published in a scientific/medical journal and be easily accessed through electronic means or retrieved through a printed library version. Case series/reports, animal studies, or articles published on independent websites were excluded. Modifications in methodology that greatly deviate from the conventional protocol and interventions that could interfere with the results were also weighed up. Statistical analysis methods and presented data were also taken into account, in terms of providing sensitivity/specificity and PPV/NPV or otherwise reporting the appropriate data sets in order to calculate these values and determine the resulting diagnostic 


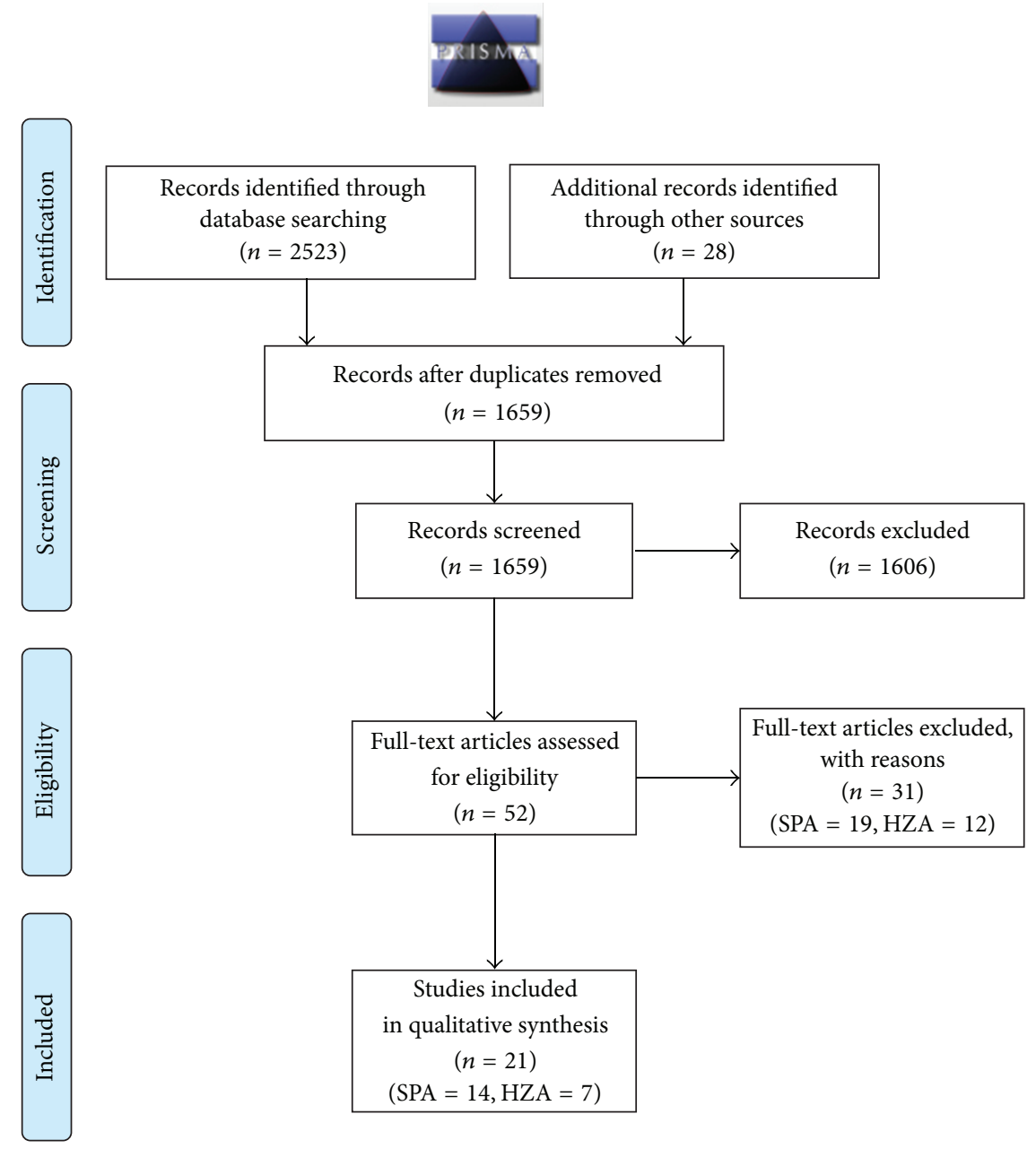

FIGURE 1: PRISMA flow diagram of study inclusion process.

accuracy, since these are the parameters that define the validity of diagnostic tests according to ESHRE [9]. Eligible studies were excluded for the following reasons: protocols including pre-treatments or interventions that could affect the results, studies with other than fertilization rate as a primary outcome or through natural conception and other assisted conception techniques (intrauterine inseminationIUI, intracytoplasmic sperm injection-ICSI, subzonal sperm insemination-SUZI), and essential data for diagnostic accuracy not shown and grouped data for true positives/negatives and false- positives/negatives missing. Where applicable, data was analyzed as published by each study group; reevaluations were not included. Reported oocyte immaturity and laboratory/equipment failure were disregarded if these results were already incorporated in the presented data. The outcome measure was fertilization outcome through IVF compared to SPA/HZA results with statistical significance by reporting specificity, sensitivity, PPV, and NPV values for diagnostic power of the assays.

\section{Results}

The initial search yielded 2551 potentially relevant studies that were reduced to 1659 by removing duplicates (Figure 1).
All titles and abstracts were screened to exclude irrelevant publications, resulting in 52 potentially eligible studies. The full-text articles were assessed, and 21 manuscripts (14 on SPA and 7 on HZA) were identified to provide data corresponding to the research question [23-43]. Characteristics of the studies included publication date, study design, and size as well as patient characteristics and experimental interventions (capacitation medium type and pre-incubation lengths/oocyte source and preservation means) which are presented in Tables 1(a) and 1(b), for SPA and HZA, respectively. Assay methodology researched in this review has been presented in supplemental Figures 1 and 2, for SPA and HZA, respectively. There were no limitations in participants' age, and fertility status was either unknown or defined through conventional semen analysis (hereby reported as normal/ abnormal semen parameters), while control groups consisted of sperm samples from donors with proven fertility and normal semen parameters. A summary of assay and fertilization rate cutoff values, along with sensitivity/specificity, PPV/NPV, are presented in Tables 2 and 3, for SPA and HZA, respectively. Statistical values were either extracted from the manuscripts or calculated through the reported values to provide the diagnostic accuracy parameters for each test. 
TABLE 1: (a) Experimental parameters of studies investigating the validity of SPA. (b) Experimental parameters of studies investigating the validity of HZA.

(a)

\begin{tabular}{|c|c|c|c|c|c|c|c|}
\hline Study group & Publication date & Study design & $n$ & $\begin{array}{l}\text { Normal SP } \\
\quad(n)\end{array}$ & $\begin{array}{l}\text { Abnormal SP } \\
(n)\end{array}$ & Capacitation medium & Pre-incubation period \\
\hline $\begin{array}{l}\text { Margalioth et al. } \\
\text { [23] }\end{array}$ & 1983 & Prospective & 20 & 20 & 0 & NR & NR \\
\hline Wolf et al. [24] & 1983 & Prospective & 24 & 24 & 0 & $\begin{array}{l}\text { Ham's F-10 with 7,5\% } \\
\text { maternal serum }\end{array}$ & $3 \mathrm{hrs}$ \\
\hline Foreman et al. [25] & 1984 & Prospective & 22 & 14 & 8 & Earle’s with 8\% HSA & $3 \mathrm{hrs}$ \\
\hline $\begin{array}{l}\text { Ausmanas et al. } \\
\text { [26] }\end{array}$ & 1985 & Prospective & 54 & 42 & 12 & $\begin{array}{c}\text { BWW with } 35 \mathrm{mg} / \mathrm{mL} \\
\text { HSA }\end{array}$ & $5 \mathrm{hrs}$ \\
\hline Belkien et al. [27] & 1985 & Prospective & 29 & 23 & 6 & $\begin{array}{l}\text { Ham's F-10 with } 10 \% \\
\text { maternal serum }\end{array}$ & $6 \mathrm{hrs}$ \\
\hline $\begin{array}{l}\text { Margalioth et al. } \\
\text { [28] }\end{array}$ & 1986 & Prospective & 134 & 124 & 10 & $\begin{array}{l}\text { mBWW with } \\
35 \mathrm{mg} / \mathrm{mL} \text { HSA }\end{array}$ & $18 \mathrm{hrs}$ \\
\hline Corson et al. [29] & 1987 & Prospective & 30 & 17 & 13 & $\begin{array}{c}\text { BWW with } 5 \mathrm{mg} / \mathrm{mL} \\
\text { HSA }\end{array}$ & $18-20 \mathrm{hrs}$ \\
\hline Kruger et al. [30] & 1988 & Retrospective & 84 & NR & NR & NR & NR \\
\hline Coetzee et al. [31] & 1989 & Prospective & 71 & NR & NR & BWW & $18-20 \mathrm{hrs}$ \\
\hline Ibrahim et al. [32] & 1989 & Prospective & 59 & 35 & 24 & $\begin{array}{l}\text { BWW with } \\
3.5 \mathrm{mg} / \mathrm{mL} \text { HSA }\end{array}$ & $3 \mathrm{hrs}$ \\
\hline $\begin{array}{l}\text { Nahhas and } \\
\text { Blumenfeld [33] }\end{array}$ & 1989 & Prospective & 31 & 27 & 4 & $\begin{array}{c}\text { BWW with } 36 \mathrm{mg} / \mathrm{mL} \\
\text { HSA }\end{array}$ & $18 \mathrm{hrs}$ \\
\hline \multirow[t]{2}{*}{ McClure et al. [34] } & 1990 & Prospective & 19 & 10 & 9 & $\begin{array}{c}\text { BWW with } 6 \mathrm{mg} / \mathrm{mL} \\
\text { HSA }\end{array}$ & $18 \mathrm{hrs}$ \\
\hline & & & & & & $\begin{array}{l}0.4 \mathrm{~mL} \text { of Human } \\
\text { Follicular Fluid }\end{array}$ & $0.5 \mathrm{hrs}$ \\
\hline Soffer et al. [35] & 1992 & Follow-up & 241 & NR & NR & TEST yolk buffer & $18-22 \mathrm{hrs}$ \\
\hline Freeman et al. [36] & 2001 & Prospective & 216 & NR & NR & $\begin{array}{l}\text { BWW with TEST } \\
\text { yolk buffer }\end{array}$ & $18-20 \mathrm{hrs}$ \\
\hline
\end{tabular}

Abbreviations: $n$ (number of participants/patients), SP (Semen Parameters), BWW (Biggers, Whitten and Whittingham medium), HSA (Human Serum Albumin), NR (Not Reported).

(b)

\begin{tabular}{|c|c|c|c|c|c|c|c|}
\hline Study group & Publication date & Study design & $n$ & Normal SP $(n)$ & Abnormal SP $(n)$ & Oocyte source & $\begin{array}{c}\text { Oocyte } \\
\text { preservation }\end{array}$ \\
\hline $\begin{array}{l}\text { Oehninger et al. } \\
\text { [37] }\end{array}$ & 1989 & Prospective & 28 & 17 & 11 & SR ovarian tissue & Salt storage \\
\hline $\begin{array}{l}\text { Oehninger et al. } \\
\text { [38] }\end{array}$ & 1991 & Prospective & 37 & 15 & 22 & IVF patient donation & Salt storage \\
\hline $\begin{array}{l}\text { Oehninger et al. } \\
{[39]}\end{array}$ & 1992 & Prospective & 44 & 31 & 13 & SR ovarian tissue & Salt storage \\
\hline Franken et al. [40] & 1993 & Prospective & 112 & 58 & 54 & $\begin{array}{l}\text { SR ovarian tissue and } \\
\text { IVF patient donation }\end{array}$ & Salt storage \\
\hline Franken et al. [41] & 1993 & Prospective & 48 & 28 & 20 & $\begin{array}{l}\text { SR ovarian tissue and } \\
\text { IVF patient donation }\end{array}$ & Salt Storage \\
\hline Gamzu et al. [42] & 1994 & Prospective & 65 & 47 & 18 & IVF patient donation & Salt Storage \\
\hline $\begin{array}{l}\text { Oehninger et al. } \\
\text { [43] }\end{array}$ & 1997 & Prospective & 196 & 138 & 58 & SR ovarian tissue & Salt storage \\
\hline
\end{tabular}

Abbreviations: $n$ (number of participants/patients), SP (Semen Parameters), SR (Surgically Recovered).

The addressed usefulness and validity of SPA were presented with major discrepancies in protocols, statistical analysis, and outcome. The inconsistencies in capacitation medium type and incubation lengths in SPA methodology are illustrated in Table 1(a). Protocol variation across studies was evident in the range of SPA cutoff values used (Table 2), final sperm concentration for the assay ranging from 0.4 to $20 \times 10^{6} / \mathrm{mL}$, in contrast to the suggested $5 \times 10^{6}$ by the WHO manuals [44] and sperm preparation methods $[45,46]$. Second and third generation SPA promoted the addition of 
TABLE 2: SPA and IVF outcome and summary of diagnostic accuracy findings.

\begin{tabular}{|c|c|c|c|c|c|c|}
\hline Study group, date & SPA threshold (\%) & Fertilization rate cut-off (\%) & Sensitivity & Specificity & PPV & NPV \\
\hline Margalioth et al., 1983 [23] & $\geq 20$ & $>0$ & $100 \%$ & $70 \%$ & $77 \%$ & $100 \%$ \\
\hline Wolf et al., 1983 [24] & $\geq 10$ & $>0$ & $88 \%$ & $0 \%$ & $68 \%$ & $0 \%$ \\
\hline Foreman et al., 1984 [25] & $>10$ & $>0$ & $67 \%$ & $53 \%$ & $18 \%$ & $91 \%$ \\
\hline Ausmanas et al., 1985 [26] & $>15$ & $>0$ & $73 \%$ & $33 \%$ & $95 \%$ & $7 \%$ \\
\hline Belkien et al., 1985 [27] & $>15$ & $>0$ & $87 \%$ & $17 \%$ & $80 \%$ & $25 \%$ \\
\hline Margalioth et al., 1986 [28] & $>20$ & $>0$ & $94 \%$ & $57 \%$ & $85 \%$ & $78 \%$ \\
\hline Corson et al., 1987 [29] & $\geq 11$ & $>0$ & $52 \%$ & $100 \%$ & $100 \%$ & $39 \%$ \\
\hline Kruger et al., 1988 [30] & $>10$ & $>0$ & $59 \%$ & $62 \%$ & $82 \%$ & $33 \%$ \\
\hline Coetzee et al., 1989 [31] & $>10$ & $>0$ & $65 \%$ & $85 \%$ & $95 \%$ & $35 \%$ \\
\hline Ibrahim et al., 1989 [32] & $>17$ & $>0$ & $74 \%$ & $84 \%$ & NR & NR \\
\hline $\begin{array}{l}\text { Nahhas and Blumenfeld, } \\
1989 \text { [33] }\end{array}$ & $>20$ & $>0$ & $100 \%$ & $44 \%$ & $81 \%$ & $100 \%$ \\
\hline McClure et al. 1990 [34] & $>10$ & $>0$ & $93 \%$ & $75 \%$ & $93 \%$ & $75 \%$ \\
\hline Soffer et al., 1992 [35] & $>20$ & $>0$ & $96 \%$ & NR & $82 \%$ & $74 \%$ \\
\hline Freeman et al., 2001 [36] & $\geq 20$ & $>50$ & $70 \%$ & $99 \%$ & $98 \%$ & $84 \%$ \\
\hline
\end{tabular}

Abbreviations: PPV (positive predictive value), NPV (negative predictive value), and NR (not reported).

TABLE 3: HZA and IVF outcome and summary of diagnostic accuracy findings.

\begin{tabular}{|c|c|c|c|c|c|c|}
\hline Study group, date & HZI threshold (\%) & Fertilization rate cutoff (\%) & Sensitivity & Specificity & PPV & NPV \\
\hline Oehninger et al., 1989 [37] & $>36$ & $>65$ & $83 \%$ & $95 \%$ & $83 \%$ & $95 \%$ \\
\hline Oehninger et al., 1991 [38] & $>36$ & $>65$ & $80 \%$ & $100 \%$ & $100 \%$ & $85 \%$ \\
\hline \multirow[t]{2}{*}{ Oehninger et al., 1992 [39] } & $>35$ & $>65$ & $100 \%$ & $61 \%$ & $79 \%$ & $100 \%$ \\
\hline & & $>0$ & $90 \%$ & $57 \%$ & $82 \%$ & $73 \%$ \\
\hline Franken et al., 1993 [40] & $>30$ & $>50$ & $84 \%$ & $72 \%$ & $85 \%$ & $70 \%$ \\
\hline Franken et al., 1993 [41] & $>30$ & $>55$ & $75 \%$ & $75 \%$ & $81 \%$ & $68 \%$ \\
\hline Gamzu et al., 1994 [42] & $>23$ & $>66$ & $100 \%$ & $94 \%$ & $85 \%$ & $100 \%$ \\
\hline Oehninger et al., 1997 [43] & $>30$ & $>60$ & $93 \%$ & $73 \%$ & $85 \%$ & $87 \%$ \\
\hline
\end{tabular}

Abbreviations: HZI (hemizona index), PPV (positive predictive value), and NPV (negative predictive value).

mild enhancers, hyperosmotic medium, albumin, follicular fluid, TEST yolk buffer, calcium ionophore, progesterone, and pentoxyfline, at variable concentrations and preincubation lengths ( 0.5 to 22 hours), so as to enhance sperm parameters and achieve capacitation prior to assay. This enabled the examination of different approaches that could contribute in assay improvement but rendered great interlaboratory inconsistency and ambiguous results over efficiency. In addition, the majority of studies did not address neither female factor and potential oocyte deficiency that could affect IVF outcome, nor the period of abstinence of the participants.

Analysis of data indicated 7 studies to have presented SPA results that correlate well with IVF outcome [23, 28, $32-36]$, indicating this assay to be a valuable prognostic tool when evaluating sperm function. The drawback is that these studies fluctuate extensively in specificity (44-100\%) with wide variation in preparation methods, capacitation medium, preincubation lengths, variation in SPA cutoff values (10-20\%), and fertilization rate cutoff values. Others have suggested a relative predictive value of SPA at some level, but data analysis failed to present the anticipated values to establish this assay as a valuable predictor of fertilization outcome [24, 26, 29]. Conversely, the clinical relevance of SPA has been debated, and significant false-positive/negative rates were demonstrated $[25,27,31]$, suggesting that this assay is of limited predictive value and unreliable for IVF. Furthermore, Kruger et al. [30] stated that, although a negative SPA might indicate male factor infertility, this assay could not be justified as a better predictor than sperm morphology assessment in IVF outcome. Other studies that investigated efficacy on a different setting than the one examined on this review could not validate SPA as a better predictor than computer assisted semen analysis (CASA), manual motility scoring, or even conventional semen analysis $[47,48]$.

The predictive power of HZA was reviewed along with possible drawbacks of this technique and protocol standardization. As summarized by Yao et al. [49], some degree of variation in HZA outcome could be induced by oocyte source and preservation method, sperm concentration and preparation method, base medium and aspiration pipette diameter, and oocyte microdissection method. Consequently, some modifications of the initial protocol were proposed [16, 17, 50-52]. 
Initial testing with a range of HZA cutoff values (15-36\%) did not enable the standardization of a baseline value; however, some range reduction was later achieved. Patient characteristics are summarized in Table 1(b), in the form of normal/abnormal sperm parameters, and the variable oocyte sources exploited by different studies are demonstrated. There are variable but limited sources of zonae, including ovarian tissue from surgical cases or cadavers and noninseminated/unfertilized oocytes donated by IVF patients. In an attempt to provide an alternative oocyte source for the assay, species like gibbons and gorillas were investigated in terms of the oocyte ability to interact with human spermatozoa [5355], but this process is highly species specific, and none of these options was further explored. Subsequently, a major drawback is the limited availability of human oocytes, a problem which could be circumvented with the possible availability of biologically active recombinant human zona as a substitute, along with other chemical/biological candidates [56-59].

The predictive value of HZA has been extensively investigated in terms of IVF outcome and the sum of the studies included in this review served to validate this assay as a predictor of fertilizing capacity [37-43]. A variable range of HZI threshold values (23-36\%) was noted (most studies employed either $30 \%$ or $36 \%$ ), and there was some variation in fertilization rate cutoff values (50-66\%) and in oocyte source, but the analysis of the published data of the selected studies for this review consistently reported high sensitivity and specificity, with concomitantly high PPV and NPV (Table 3). Increased diagnostic accuracy values demonstrated throughout indicate that in an IVF setting HZA results are specific to fertility potential, establishing whether spermatozoa of the male under investigation are functionally competent to interact with the oocyte and complete a sequence of actions essential for fertilization.

\section{Discussion}

Analysis of the published evidence on the preselected sperm function tests validity demonstrated inconsistent results on SPA's role in male fertility evaluation, while HZA was consistently efficient in predicting IVF outcome. When reviewing data on SPA, considerable variation was noted in diagnostic accuracy values with wide sensitivity (52-100\%), specificity (0-100\%), and PPV (18-100\%) and NPV (0-100\%) together with fluctuation and notable differentiation in methodology and cutoff values employed by each group. On the other hand, HZA methodology was overall consistent with minor variation in cutoff values and oocyte source, while data analysis reported strong correlation of HZA results with IVF outcome. These studies consistently reported high HZA sensitivity (75-100\%), good specificity (57-100\%), and high PPV (79-100\%) and NPV (68-100\%).

On the level of association of SPA with IVF outcome [23-36], some studies reported good correlation, and others could not validate any apparent connection, whereas the rest determined a relative predictive value under specific conditions. Unsurprisingly, these contrasting views have brought about uncertainty over the power of this tool in male fertility assessment. The relevant meta-analyses $[60,61]$ confirmed the heterogeneity among studies and overall low predictive power of SPA on IVF outcome with increased falsepositives and, therefore, reduced specificity. In particular, Mol et al. [60] demonstrated significant heterogeneity of the assay with low sensitivity (37\%) and, interestingly, high specificity (95\%) and concluded that SPA is an inadequate assay for selecting patients for IVF treatment. Similarly, Oehninger et al. [61] reported a high false-positive rate and thus reduced specificity of SPA as a predictor of fertilization, corroborated also by the European Society of Human Reproduction and Embryology [62], which accordingly recommended its abandonment at that point. Compared with much simpler approaches, such as sperm motility assessment, strict morphology, or even conventional seminology, SPA did not appear to be superior $[30,47,48]$. SPA is still a widely applied research tool as it examines certain functional aspects, but it is currently of limited value in clinical practice, since it does not contribute significant information in male fertility status. Standardised protocols may provide diagnostic information with greater precision in terms of sensitivity and specificity, as well as replicating gamete physiological interaction more accurately, and this is in agreement with Oehninger et al. [61] suggesting that SPA methodology should be revisited to produce a consensus protocol.

HZA has undergone little variation since its introduction and consistency of methodology are preserved to a great extent across IVF laboratories. A considerable limitation in the applicability of the assay is the restricted reserve of human oocytes [42], although the prospective utilization of biologically active recombinant human zona pellucida could circumvent it. Furthermore, the complexity of performing oocyte dissection into two equal hemispheres requires high accuracy to avoid the potential risk of differentially exposed area between control and test samples. Finally, ethical concerns arise over the extent of the allowed gamete interaction during sperm function assays, although HZA-in contrast to the CZB-exploits oocytes devoid of ooplasm and its application could nullify any points of consideration.

In our review, HZA appeared to be an important marker of spermatozoa fertilizing capacity. It correlated well with IVF outcome, as it demonstrated better sensitivity/specificity and positive/negative predictive power than SPA, in agreement with the findings of a previous meta-analysis [61], reporting high PPV and NPV and consistently low false-negative rates. The noted result of homogeneity of the included studies, together with the subtle methodology variation between laboratories, indicates that HZA is a reliable candidate to assess sperm function capacity. Males with normal or slightly abnormal semen profiles could benefit by this intervention and be evaluated prior to referral to assisted reproduction. This assay provides essential information on several aspects of sperm function and could be used in a sequential fashion with semen analysis and potentially other bioassays in an ART setting.

The limitations of the current review reflect the corresponding limitations of the individual studies. Although numerous records were originally retrieved during our 
search, studies that used different assisted conception methods such as intrauterine insemination (IUI) or ICSI, or with primary outcomes such as live birth rate, or pregnancy rate were excluded to preserve consistency over the examined parameters defining the outcome of each study. This was also considered in the context of the exact process that reflects functional capacity of the spermatozoon where fertilization is the outmost measure compared to pregnancy and live birth where other factors such as chromosomal integrity of the spermatozoon, embryo abnormalities, and pregnancy complications could potentially have a major impact on these outcomes. In the same context, protocols including pretreatments or interventions that could exogenously affect sperm function were excluded, in an attempt to signify the value of these tests in a clear-cut manner. Most of the included studies were published over two decades ago-when these techniques were considered breakthrough and were extensively investigated-except that at this period publication criteria differed significantly, leading to missing crucial information and statistical reporting methods relying on the authors' discretion. This limited our ability to explore the different approaches of the assay protocols, the efficiency of the interventions used and participant characteristics to assist direct comparison and possible attempts to communicate with the authors for further clarification were abandoned due to the restricted contact information published at that point. Moreover, this generated the necessity to recalculate diagnostic accuracy values wherever the data sets were available and led to the exclusion of studies that did not report the appropriate statistical values or corresponding data sets. A possible limitation could be attributed with regard to cost efficiency of the particular sperm function tests in practise, but this parameter was not included in our initial goals towards investigation as the core purpose of this review was to clarify the efficiency of these tests in terms of IVF outcome. A previously published review and meta-analysis [61] explored various sperm function tests in a similar manner, although this group did not include the sum of the studies reported here and no direct comparison was performed between these two tests to clarify their definite validity on male fertility investigation. Unavoidably, a number of studies coincide with Oehninger et al. [61] however, calculation of diagnostic accuracy values revealed some degree of discordance in terms of the reported specificity, sensitivity, and PPV and NPV.

Current clinical opinion relies mostly on conventional semen parameters upon deciding wether to allow natural conception or to refer for IVF treatment and, quite frequently, without identifying male fertility potential or any underlying pathology. Semen analysis is sometimes subject to the evaluators' individual judgement and has been implicated to exert limited predictive value in determining sperm function and fertility potential, as demonstrated by the noted discrepancies in cases when men with normal semen parameters have repeated fertilization failure, while men with very abnormal semen characteristics have fathered children. Some fertility clinics incorporate sperm function tests in their advanced investigations, although their clinical significance has been undermined by the introduction of intracytoplasmic sperm injection (ICSI), a technique that effectively circumvents the need for functioning sperm. However, consideration should be given to ethical and biological aspects; should natural selection be allowed to take place avoiding the invasive micromanipulation techniques that mostly rely on morphology in deciding which spermatozoon is the most fit to fertilize an oocyte.

Impaired spermatozoa interaction with the zona pellucida and oocyte investments has been classified amongst the principal causes of fertilization failure, and, in this context, our data analysis highlighted the validity and high predictive value of HZA; this result situates this assay within the criteria for plausibility and applicability of diagnostic tests. From a clinical perspective, this technically demanding technique requires an affirmed oocyte source, or the commercial availability of biologically active human recombinant zona pellucida, to confront limited oocyte availability. In contrast, SPA failed to produce uniform results on IVF outcome prediction, and this finding along with protocol inconsistency, indicate, that it is far from being an absolute test for male fertility potential. However, more effort should be devoted in optimizing and reevaluating this technique as a great advantage of SPA is that this assay utilizes hamster oocytes are more readily available than human and clinical application of SPA could possibly be more realistic than implementing an assay that requires resources which are currently limited.

Ideally, sperm function tests should incorporate a wide array of functions in a single assay; however, evidence published to date indicates an apparent dominance of HZA in the context of clinical significance. Following technical optimization and establishment of a reliable oocyte source or analogue availability, HZA could be applied in clinical practice and could be incorporated as part of a range of tests for the profiling of gamete functionality. Future technological advances could promote further improvement of sperm function tests or even the introduction of a single, conclusive test on multiple functional aspects for rapid and accurate diagnosis and optimal clinical management.

\section{Conflict of Interests}

All authors declare no conflict of interests.

\section{Authors' Contribution}

P. Vogiatzi conceived the idea of the study, contributed to study design, critical evaluation of the studies, extraction of data, and interpretation of the findings, performed analysis, drafted the article, and gave the final approval of the version to be published. C. Chrelias contributed to study design and critical evaluation of the studies, revised the paper critically for important intellectual content, and gave the final approval of the version to be published. D. J. Cahill contributed to the idea of the study and study design, drafted the paper, revised the paper critically for important intellectual content, and gave final approval of the version to be published. M. Creatsa contributed to study design, critical evaluation of the studies, and extraction and interpretation of data and gave final approval of the version to be published. 
N. Vrachnis contributed to study design, critical evaluation of the studies, and extraction and interpretation of data and gave the final approval of the version to be published. $\mathrm{Z}$. Iliodromiti contributed to study design, critical evaluation of the studies, and extraction and interpretation of data, and gave final approval of the version to be published. D. Kassanos contributed to study design, critical evaluation of the studies, and extraction and interpretation of data, and gave the final approval of the version to be published. C. Siristatidis initiated the methodology, contributed to study design, critical evaluation of the studies, and extraction and interpretation of data, drafted the paper, revised the paper critically for important intellectual content and gave final approval of the version to be published.

\section{References}

[1] G. Dohle, A. Jungwirth, G. Colpi, A. Giwerman, T. Diemer, and T. Hargreave, Guidelines on Male Infertility, European Association of Urology, 2007, http://www.uroweb.org/fileadmin/ user_upload/Guidelines/13_Male_Infertility_2007.pdf.

[2] I. D. Sharlip, J. P. Jarow, A. M. Belker et al., "Best practice policies for male infertility," Fertility and Sterility, vol. 77, no. 5, pp. 873$882,2002$.

[3] P. Thonneau, S. Marchand, A. Tallec et al., "Incidence and main causes of infertility in a resident population $(1,850,000)$ of three French regions (1988-1989)," Human Reproduction, vol. 6, no. 6, pp. 811-816, 1991.

[4] E. Carlsen, A. Giwercman, N. Keiding, and N. E. Skakkebaek, "Evidence for decreasing quality of semen during past 50 years," The British Medical Journal, vol. 305, no. 6854, pp. 609-613, 1992.

[5] S. H. Swan, E. F. Elkin, and L. Fenster, "Have sperm densities declined? A reanalysis of global trend data," Environmental Health Perspectives, vol. 105, no. 11, pp. 1228-1232, 1997.

[6] S. H. Swan, E. P. Elkin, and L. Fenster, "The question of declining sperm density revisited: an analysis of 101 studies published 1934-1996," Environmental Health Perspectives, vol. 108, no. 10, pp. 961-966, 2000.

[7] D. J. Handelsman and T. G. Cooper, "Falling sperm counts and global estrogenic pollution: what have we learned over 20 years?" Asian Journal of Andrology, vol. 15, no. 2, pp. 159-161, 2013.

[8] C. L. R. Barratt, L. Björndahl, R. Menkveld, and D. Mortimer, "ESHRE special interest group for andrology basic semen analysis course: a continued focus on accuracy, quality, efficiency and clinical relevance," Human Reproduction, vol. 26, no. 12, pp. 3207-3212, 2011.

[9] P. G. Crosignani and B. L. Rubin, "Optimal use of infertility diagnostic tests and treatments," Human Reproduction, vol. 15, no. 3, pp. 723-732, 2000.

[10] Male Infertility Best Practice Policy Committee of the American Urological Association and Practice Committee of the American Society for Reproductive Medicine, "Report on optimal evaluation of the infertile male," Fertility and Sterility, vol. 86, no. 5, supplement, pp. S202-S209, 2006.

[11] K. P. Nallella, R. K. Sharma, N. Aziz, and A. Agarwal, "Significance of sperm characteristics in the evaluation of male infertility," Fertility and Sterility, vol. 85, no. 3, pp. 629-634, 2006.
[12] S. E. M. Lewis, "Is sperm evaluation useful in predicting human fertility?” Reproduction, vol. 134, no. 1, pp. 31-40, 2007.

[13] R. Yanagimachi, H. Yanagimachi, and B. J. Rogers, "The use of zona free animal ova as a test system for the assessment of the fertilizing capacity of human spermatozoa," Biology of Reproduction, vol. 15, no. 4, pp. 471-476, 1976.

[14] D. Y. Liu, A. Lopata, W. I. H. Johnston, and H. W. G. Baker, "A human sperm-zona pellucida binding test using oocytes that failed to fertilize in vitro," Fertility and Sterility, vol. 50, no. 5, pp. 782-788, 1988.

[15] L. J. Burkman, C. C. Coddington, D. R. Franken, T. F. Kruger, Z. Rosenwaks, and G. D. Hodgen, "The hemizona assay (HZA): development of a diagnostic test for the binding of human spermatozoa to the human hemizona pellucida to predict fertilization potential," Fertility and Sterility, vol. 49, no. 4, pp. 688-697, 1988.

[16] D. R. Franken, L. J. Burkman, S. C. Oehninger et al., "Hemizona assay using salt-stored human oocytes: evaluation of zona pellucida capacity for binding human spermatozoa," Gamete Research, vol. 22, no. 1, pp. 15-26, 1989.

[17] D. R. Morroll, B. A. Lieberman, and P. L. Matson, "Use of human zonae from cryopreserved oocytes in a test to assess the binding capacity of human spermatozoa," International Journal of Andrology, vol. 16, no. 2, pp. 97-103, 1993.

[18] D. Y. Liu and H. W. G. Baker, "Defective sperm-zona pellucida interaction: a major cause of failure of fertilization in clinical in-vitro fertilization," Human Reproduction, vol. 15, no. 3, pp. 702-708, 2000.

[19] D. Y. Liu and H. W. G. Baker, "Frequency of defective spermzona pellucida interaction in severely teratozoospermia infertile men," Human Reproduction, vol. 18, no. 4, pp. 802-807, 2003.

[20] D. Y. Liu and H. W. G. Baker, "High frequency of defective sperm-zona pellucida interaction in oligozoospermic infertile men," Human Reproduction, vol. 19, no. 2, pp. 228-233, 2004.

[21] R. J. Aitken, “Sperm function tests and fertility," International Journal of Andrology, vol. 29, no. 1, pp. 69-75, 2006.

[22] A. Liberati, D. G. Altman, J. Tetzlaff et al., "The PRISMA statement for reporting systematic reviews and meta-analyses of studies that evaluate health care interventions: explanation and elaboration," PLoS Medicine, vol. 6, no. 7, Article ID e1000100, 2009.

[23] E. J. Margalioth, D. Navot, N. Laufer et al., "Zone-free hamster ovum penetration assay as a screening procedure for in vitro fertilization," Fertility and Sterility, vol. 40, no. 3, pp. 386-388, 1983.

[24] D. P. Wolf, J. E. Sokoloski, and M. M. Quigley, "Correlation of human in vitro fertilization with the hamster egg bioassay," Fertility and Sterility, vol. 40, no. 1, pp. 53-59, 1983.

[25] R. Foreman, J. Cohen, C. B. Fehilly, S. B. Fishel, and R. G. Edwards, "The application of the zona-free hamster egg test for the prognosis of human in vitro fertilization," Journal of In Vitro Fertilization and Embryo Transfer, vol. 1, no. 3, pp. 166-171, 1984.

[26] M. Ausmanas, R. W. Tureck, L. Blasco, G. S. Kopf, J. Ribas, and L. Mastroianni Jr., "The zona-free hamster egg penetration assay as a prognostic indicator in a human in vitro fertilization program," Fertility and Sterility, vol. 43, no. 3, pp. 433-437, 1985.

[27] L. Belkien, J. Bordt, C. W. Freischem, R. Hano, U. A. Knuth, and E. Nieschlag, "Prognostic value of the heterologous ovum penetration test for human in vitro fertilization," International Journal of Andrology, vol. 8, no. 4, pp. 275-284, 1985. 
[28] E. J. Margalioth, D. Navot, N. Laufer, A. Lewin, R. Rabinowitz, and J. G. Schenker, "Correlation between the zona-free hamster egg sperm penetration assay and human in vitro fertilization," Fertility and Sterility, vol. 45, no. 5, pp. 665-670, 1986.

[29] S. L. Corson, F. R. Batzer, K. J. Go et al., "Correlations between the human sperm-hamster egg penetration assay and in vitro fertilization results," Journal of Reproductive Medicine for the Obstetrician and Gynecologist, vol. 32, no. 12, pp. 879-887, 1987.

[30] T. F. Kruger, R. J. Swanson, M. Hamilton et al., "Abnormal sperm morphology and other semen parameters related to the outcome of the hamster oocyte human sperm penetration assay," International Journal of Andrology, vol. 11, no. 2, pp. 107113, 1988.

[31] K. Coetzee, T. F. Kruger, R. Menkveld, R. J. Swanson, C. J. Lombard, and A. A. Acosta, "Usefulness of sperm penetration assay in fertility predictions," Archives of Andrology, vol. 23, no. 3, pp. 207-212, 1989.

[32] M. E. Ibrahim, M. A. A. Moussa, and H. Pedersen, "Efficacy of zona-free hamster egg sperm penetration assay as a predictor of in vitro fertilization," Archives of Andrology, vol. 23, no. 3, pp. 267-274, 1989.

[33] F. Nahhas and Z. Blumenfeld, "Zona-free hamster egg penetration assay: prognostic indicator in an IVF program," Archives of Andrology, vol. 23, no. 1, pp. 33-37, 1989.

[34] R. D. McClure, R. A. Tom, and P. V. Dandekar, "Optimizing the sperm penetration assay with human follicular fluid," Fertility and Sterility, vol. 53, no. 3, pp. 546-550, 1990.

[35] Y. Soffer, A. Golan, A. Herman, M. Pansky, E. Caspi, and R. Ron-El, "Prediction of in vitro fertilization outcome by sperm penetration assay with TEST-yolk buffer preincubation," Fertility and Sterility, vol. 58, no. 3, pp. 556-562, 1992.

[36] M. R. Freeman, A. E. Archibong, J. J. Mrotek, C. M. Whitworth, G. A. Weitzman, and G. A. Hill, "Male partner screening before in vitro fertilization: preselecting patients who require intracytoplasmic sperm injection with the sperm penetration assay," Fertility and Sterility, vol. 76, no. 6, pp. 1113-1118, 2001.

[37] S. Oehninger, C. C. Coddington, R. Scott et al., "Hemizona assay: assessment of sperm dysfunction and prediction of in vitro fertilization outcome," Fertility and Sterility, vol. 51, no. 4, pp. 665-670, 1989.

[38] S. Oehninger, L. Veeck, D. Franken, T. F. Kruger, A. Acosta, and G. D. Hodgen, "Human preovulatory oocytes have a higher sperm-binding ability than immature oocytes under hemizona assay conditions: evidence supporting the concept of "zona maturation"'” Fertility and Sterility, vol. 55, no. 6, pp. 1165-1170, 1991.

[39] S. Oehninger, D. Franken, N. Alexander, and G. D. Hodgen, "Hemizona assay and its impact on the identification and treatment of human sperm dysfunctions," Andrologia, vol. 24, no. 6, pp. 307-321, 1992.

[40] D. R. Franken, T. F. Kruger, S. Oehninger et al., "The ability of the hemizona assay to predict human fertilization in different and consecutive in-vitro fertilization cycles," Human Reproduction, vol. 8, no. 8, pp. 1240-1244, 1993.

[41] D. R. Franken, A. A. Acosta, T. F. Kruger, C. J. Lombard, S. Oehninger, and G. D. Hodgen, "The hemizona assay: its role in identifying male factor infertility in assisted reproduction," Fertility and Sterility, vol. 59, no. 5, pp. 1075-1080, 1993.

[42] R. Gamzu, L. Yogev, A. Amit, J. Lessing, Z. T. Homonnai, and H. Yavetz, "The hemizona assay is of good prognostic value for the ability of sperm to fertilize oocytes in vitro," Fertility and Sterility, vol. 62, no. 5, pp. 1056-1059, 1994.
[43] S. Oehninger, P. Kolm, M. Mahony, T. Kruger, K. Özgür, and D. Franken, "Clinical significance of human sperm-zona pellucida binding," Fertility and Sterility, vol. 67, no. 6, pp. 1121-1127, 1997.

[44] World Health Organization, WHO Laboratory Manual for the Examination of Human Semen and Sperm-Cervical Mucus Interaction, Cambridge University Press, Cambridge, UK, 1992.

[45] D. Mortimer, "Sperm preparation techniques and iatrogenic failures of in-vitro fertilization," Human Reproduction, vol. 6, no. 2, pp. 173-176, 1991.

[46] S. Y. W. Chan and M. J. Tucker, "Differential sperm performance as judged by the zona-free hamster egg penetration test relative to differing sperm penetration techniques," Human Reproduction, vol. 7, no. 2, pp. 255-260, 1992.

[47] W. C. L. Ford, K. M. Williams, S. Harrison et al., "Value of the hamster oocyte test and computerised measurements of sperm motility in predicting if four or more viable embryos will be obtained in an IVF cycle," International Journal of Andrology, vol. 24, no. 2, pp. 109-119, 2001.

[48] L. M. Ho, A. S. T. Lim, T. H. Lim, S. C. Hum, S. L. Yu, and T. F. Kruger, "Correlation between semen parameters and the hamster egg penetration test (HEPT) among fertile and subfertile men in Singapore," Journal of Andrology, vol. 28, no. 1, pp. 158-163, 2007.

[49] Y. Q. Yao, W. S. B. Yeung, and P. C. Ho, “The factors affecting sperm binding to the zona pellucida in the hemizona binding assay," Human Reproduction, vol. 11, no. 7, pp. 1516-1519, 1996.

[50] D. Y. Liu and H. W. G. Baker, "Inhibition of acrosin activity with a trypsin inhibitor blocks human sperm penetration of the zona pellucida," Biology of Reproduction, vol. 48, no. 2, pp. 340-348, 1993.

[51] D. Y. Liu and H. W. G. Baker, "A new test for the assessment of sperm-zona pellucida penetration: relationship with results of other sperm tests and fertilization in vitro," Human Reproduction, vol. 9, no. 3, pp. 489-496, 1994.

[52] M. Paul, J. P. Sumpter, and K. S. Lindsay, “The paradoxical effects of pentoxifylline on the binding of spermatozoa to the human zona pellucida," Human Reproduction, vol. 11, no. 4, pp. 814-819, 1996.

[53] J. M. Bedford, "Sperm/egg interaction: the specificity of human spermatozoa," Anatomical Record, vol. 188, no. 4, pp. 477-487, 1977.

[54] R. Yanagimachi, "Sperm-egg fusion," Current Topics in Membranes and Transport, vol. 32, pp. 3-43, 1988.

[55] D. Y. Liu, A. Lopata, P. Pantke, and H. W. G. Baker, "Horse and marmoset monkey sperm bind to the zona pellucida of saltstored human oocytes," Fertility and Sterility, vol. 56, no. 4, pp. 764-767, 1991.

[56] M. van Duin, J. E. M. Polman, I. T. M. de Breet et al., "Recombinant human zona pellucida protein $\mathrm{ZP} 3$ produced by Chinese hamster ovary cells induces the human sperm acrosome reaction and promotes sperm-egg fusion," Biology of Reproduction, vol. 51, no. 4, pp. 607-617, 1994.

[57] I. A. Brewis, R. Clayton, C. L. R. Barratt, D. P. J. Hornby, and H. D. M. Moore, "Recombinant human zona pellucida glycoprotein 3 induces calcium influx and acrosome reaction in human spermatozoa," Molecular Human Reproduction, vol. 2, no. 8, pp. 583-589, 1996.

[58] A. J. Whitmarsh, M. J. Woolnough, H. D. M. Moore, D. P. Hornby, and C. L. R. Barratt, "Biological activity of recombinant human ZP3 produced in vitro: potential for a sperm function test," Molecular Human Reproduction, vol. 2, no. 12, pp. 911-919, 1996. 
[59] K. W. Dong, T. F. Chi, Y. W. Juan et al., "Characterization of the biologic activities of a recombinant human zona pellucida protein 3 expressed in human ovarian teratocarcinoma (PA-1) cells," The American Journal of Obstetrics and Gynecology, vol. 184, no. 5, pp. 835-844, 2001.

[60] B. W. J. Mol, S. Meijer, S. Yuppa et al., "Sperm penetration assay in predicting successful in vitro fertilization: a metaanalysis," Journal of Reproductive Medicine for the Obstetrician and Gynecologist, vol. 43, no. 6, pp. 503-508, 1998.

[61] S. Oehninger, D. R. Franken, E. Sayed, G. Barroso, and P. Kolm, "Sperm function assays and their predictive value for fertilization outcome in IVF therapy: a meta-analysis," Human Reproduction Update, vol. 6, no. 2, pp. 160-168, 2000.

[62] ESHRE Andrology Special Interest Group, "Consensus workshop on advanced diagnostic andrology techniques," Human Reproduction, vol. 11, no. 7, pp. 1463-1479, 1996. 


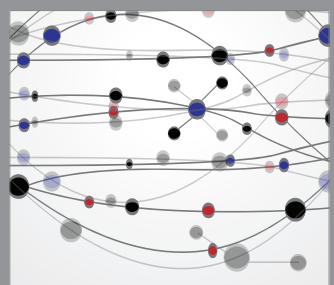

The Scientific World Journal
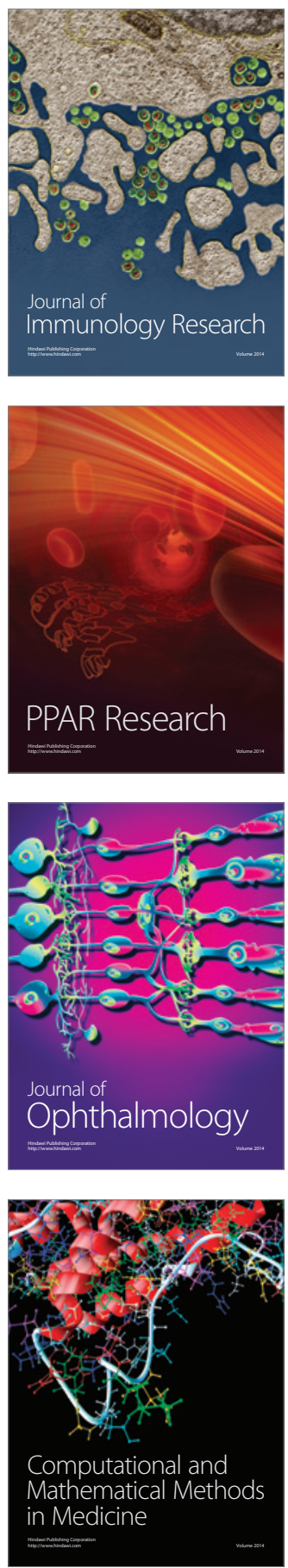

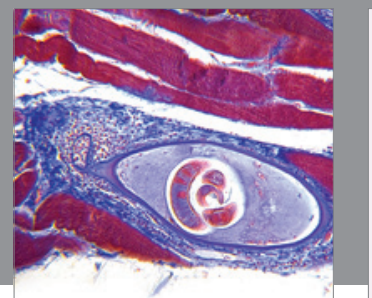

Gastroenterology

Research and Practice
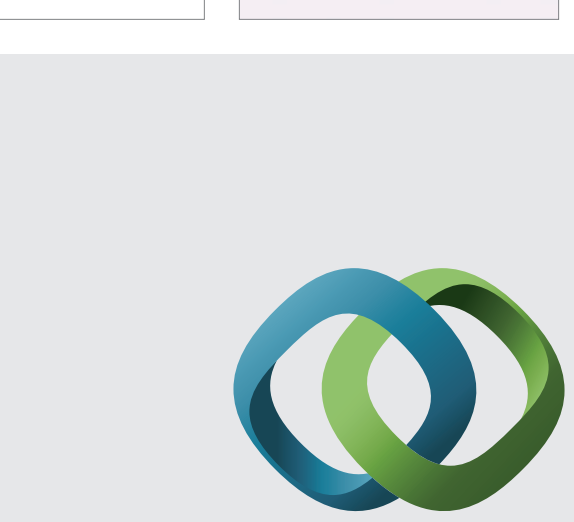

\section{Hindawi}

Submit your manuscripts at

http://www.hindawi.com
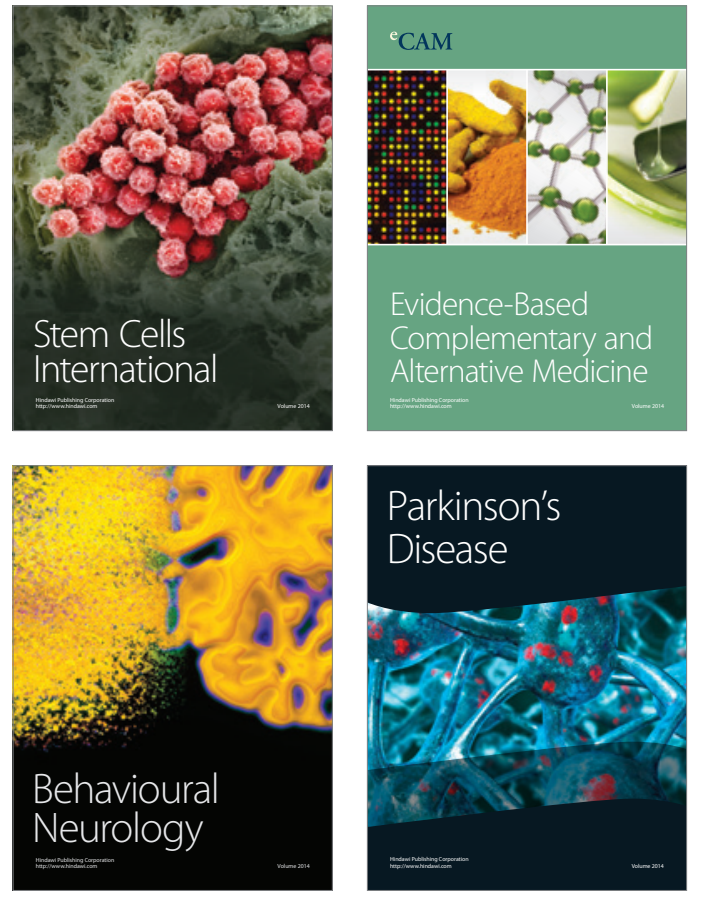
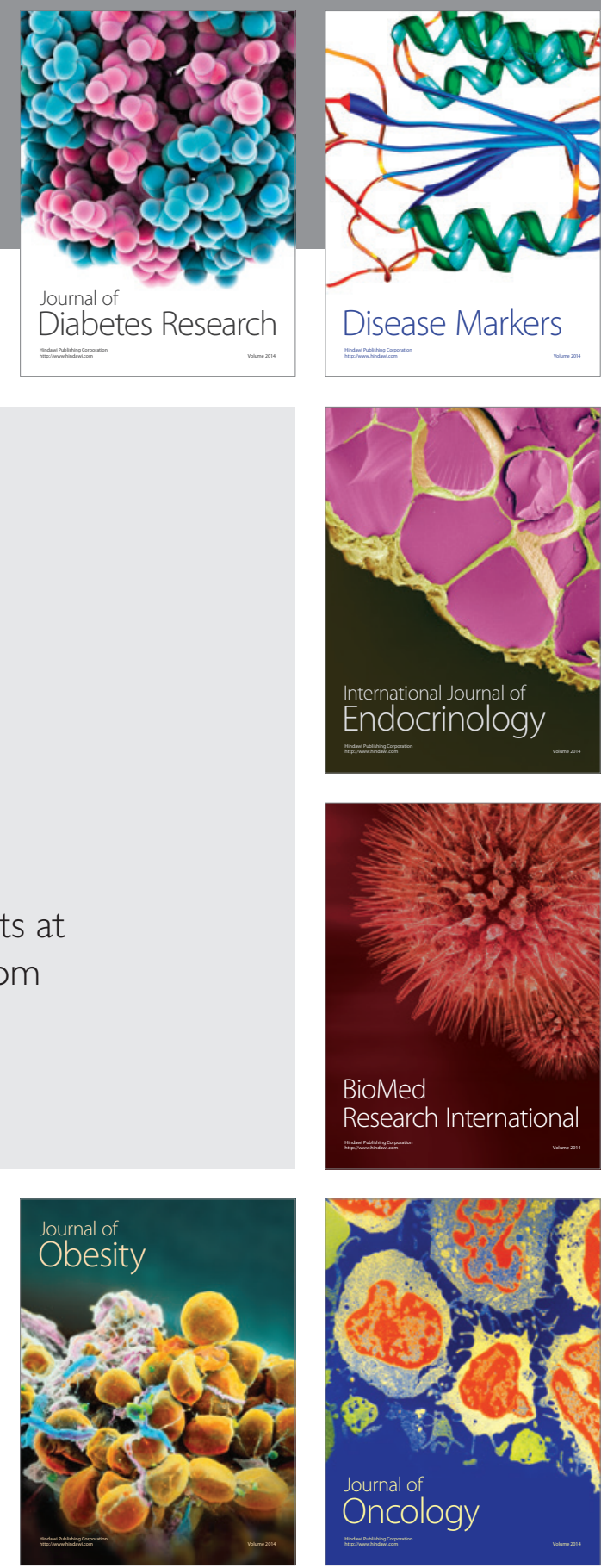

Disease Markers
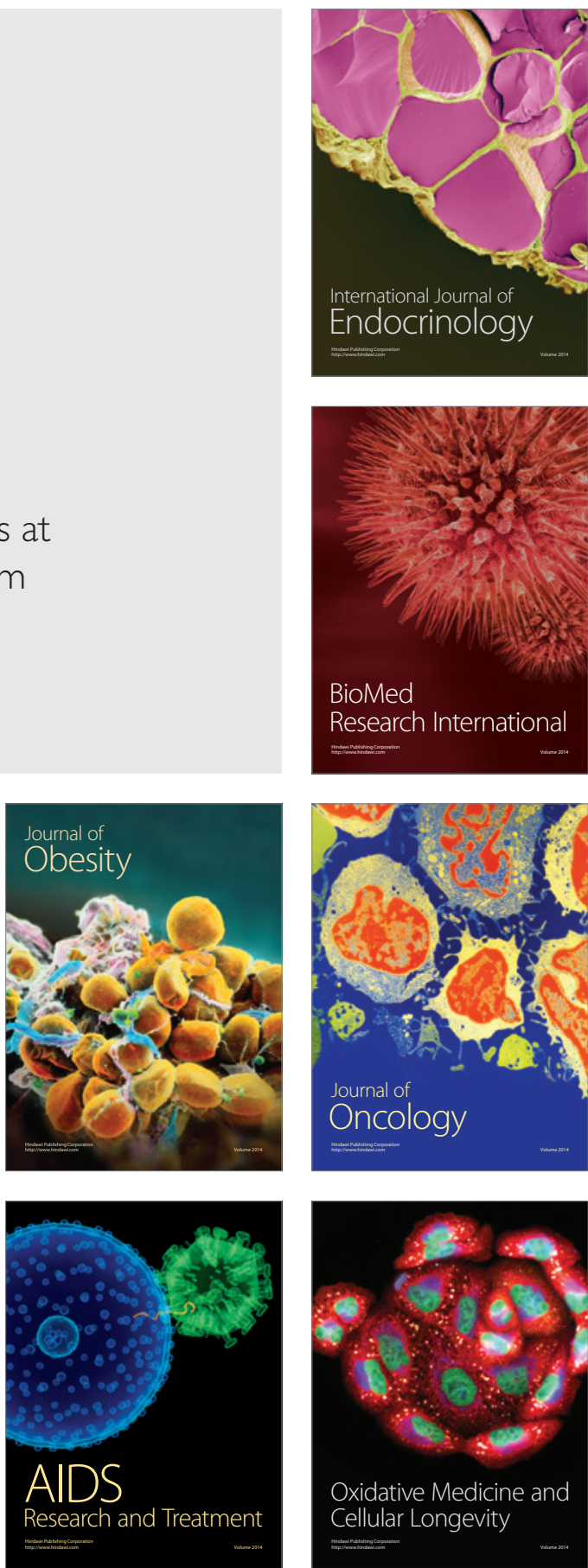